\title{
Improved Efficiency of Inductive Power Transfer in Misalignment Conditions with Multi Coil Design
}

\author{
Pranoto Budi Laksono', Mudrik Alaydrus ${ }^{2}$ \\ 1,2 Department of Electrical Engineering, Universitas Mercu Buana, Jakarta, Indonesia \\ *corresponding author, E-mail: pranotobl@gmail.com; mudrikalaydrus@mercubuana.ac.id
}

\begin{abstract}
In charging process of electric vehicle, a misalignment between the transmitter (Tx) and receiver (Rx) coupling structure decreases the efficiency of the wireless power transfer. In inductive power transfer system, misalignment reduces the effective coupling between the Tx and Rx coils. In this work, based on previous multiple coil structures, a new multi coil design in proposed to increase the efficiency of the power transfer. Here, a multi coil structure with two rectangular and four spiral coils is designed with the overall dimension of the coil structure $26.5 \mathrm{~cm} \times 36.5 \mathrm{~cm}$. The measurement shows, that for coil distance below $10.3 \mathrm{~cm}$ and a lateral misalignment of maximal $10 \mathrm{~cm}(27.4 \%)$, the efficiency of the designed multi coil structure is better compared to previous coil structures. However for larger coil distance or larger misalignment, the efficiency of the new coil structure deteriorates significantly.
\end{abstract}

\section{Introduction}

In wireless charging process of electric vehicles, the $\mathrm{Rx}$ coupling coil at the underside of the vehicle is positioned directly above the Tx coupling coil, which is normally installed permanently on the parking lot floor in the charging station. When the car is parked, it is possible that the position of the transmitter coil and the receiver coil is not in a perfect line (perfect alignment), there is a positional misalignment between the transmitter coil and the receiver coil due to the improperly parked car position causing the power transfer efficiency to decrease. This position shift is called lateral misalignment ( $\mathrm{Y}$-axis displacement or $\mathrm{X}$-axis displacement) as shown in Figure 1 [1].

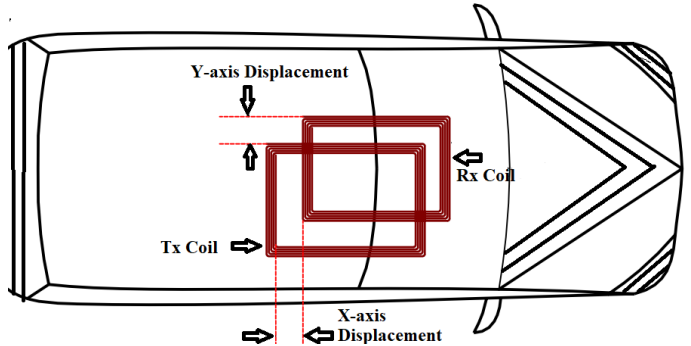

Figure 1: Coil Misalignment [1]

The orientation of the coil position is a key parameter in the design of inductive coupling systems. The lateral misalignment can be explained as the $\mathrm{Tx}$ and $\mathrm{Rx}$ coils are located in a parallel plane, separated by a distance $d$ and the shift of the central point symbolized by $\Delta$, as shown in Figure 2 [2][3].

In a small lateral misalignment condition, angular effects predominate, whereas in a large lateral misalignment, the lateral effect is the dominant ones [2]. In misalignment conditions, the power transmission is dependent on the coupling coefficient substantially [4].

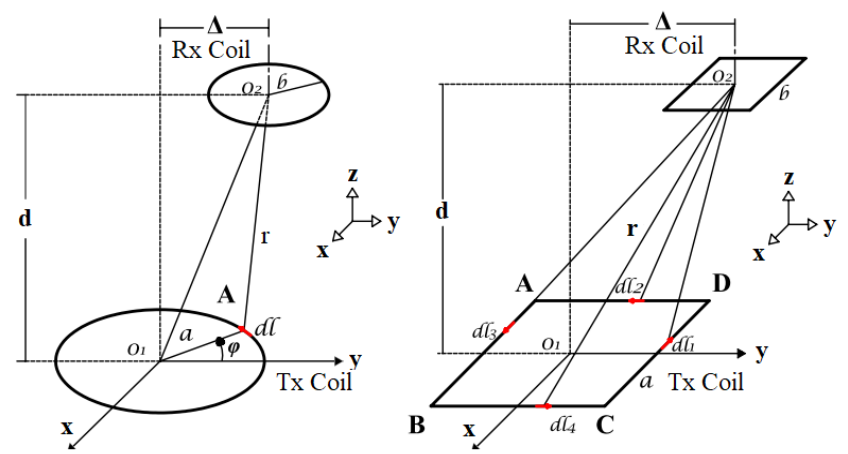

Figure 2: Lateral Misalignment [2]

This paper presents the following systematics: section 1 is introduction and background, section 2 presents the concept of Inductive Power Transfer, the geometry of the coils and the literature review. Section 3 presents the proposed coil geometry design, section 4 gives the experiment results and section 5 is conclusion and future work.

\section{Inductive Power Transfer}

Inductive power transfer is based on magnetic coupling as a power linkage from $\mathrm{Tx}$ coil to $\mathrm{Rx}$ coil. Figure 3 [5] shows a systematic model for an inductive power transfer (IPT) system. The figure reveals that some of the magnetic flux from the Tx coil intersects the Rx coil, which is located close to the Tx coil, normally less than the wavelength. The near field strength then induces a voltage across the $\mathrm{Rx}$ coil. The induced voltage can be used for charging wireless devices or another storage systems. The operating frequency of the inductive coupling is usually within the kilo-Hertz $(\mathrm{kHz})$ range. $\mathrm{Rx}$ Coil must be adjusted about the operating 
frequency of the Tx coil in order to improve fill efficiency [6] of the system. Claudio Carretero in his journal said that the working frequency at IPT ranges from a few $\mathrm{kHz}$ to radio frequency (several tens of $\mathrm{MHz}$ ) [7]. The advantages of the inductive coupling are easy to implementation, easy operation, high efficiency at close range (usually less than coil diameter), guarantee for security [8][9][10][11], low maintenance costs [10][12], reliable [10], has the ability to operate in dirty conditions [4][13].

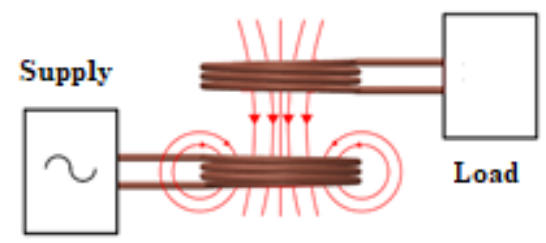

Figure 3: Inductive Coupling [5]

Generally, it can be said that IPT follows the Ampere's law and the Faraday's inductive law. The Ampere's law, or more generally the Biot Savart's law, explains the distribution of the magnetic field vector in the space, especially around the coil structures. The relationship of the direction of the current to the loop with the direction of the resulting magnetic field follows the rule of the right hand (or the screw) where the thumb shows the direction of the current, while the other finger shows the direction of the magnetic field. The Faraday's law or the induction law gives the relationship between the induced voltage and the change of the magnetic flux through the Rx coil to the time.

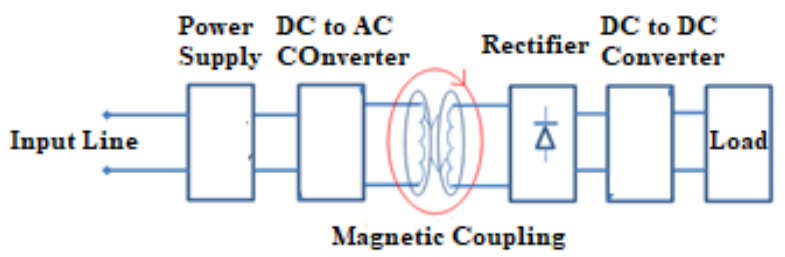

Figure 4: Block Diagram of IPT system [5]

In the IPT System (Figure 4), generally consists of three main components: power supply, two coupled coils, rectifier and DC / DC converter. The power supply produces sinusoidal current, with a frequency typically within the $\mathrm{kHz}$. The power transfer happens through inductive coupling from the Tx coil to the Rx coil. These two coils are not always identical, they can have different shapes and dimensions. The rectifier may be required to transform the high frequency $\mathrm{AC}$ voltage to DC voltage if the load to be powered is a DC load. Moreover, an additional DC/DC converter can be required to provide a regulated input DC voltage to the load [5].

There are many studies about power transfer efficiency in wireless power transfer systems. The studies used different frequencies and coil designs, and they observed the efficiency of power transfer in perfect alignment and in misalignment conditions. The difference in frequency and coil design leads to difficulties in comparing one design to the others. For example Kalwar et al [4] (2016) introduced the IPT coil design with the name QDQ (Quad D Quadrature) by combining four small circular coils placed on a large rectangle coil with a resonant frequency of $33 \mathrm{kHz}$ and a distance between $\mathrm{Tx}$ and $\mathrm{Rx}$ coils of $15 \mathrm{~cm}$. This design is made identically between Tx coils and Rx coils. Kuzey et al [14] (2017) made a coil design with rectangle shape with Tx $60 \mathrm{~cm}$ x $100 \mathrm{~cm}$ coil dimension and Rx $60 \mathrm{~cm}$ x $60 \mathrm{~cm}$ coil size with $17.702 \mathrm{kHz}$ resonance frequency.

\section{1 Coil Geometry Design}

In [4] it is mentioned that the coil of the spiral circle have a good coupling but poor tolerance at misalignment condition. The spiral circle coil have the mutual induction higher than the rectangular geometry shape at perfect alignment, but the performance during misalignment conditions, the rectangular coil is better than spiral circle. Therefore, rectangular geometry is more common in electric Vehicle applications.

Based on the Neumann's equation [15] to calculate the magnitude of the mutual inductance between two circle coils with number of turns $\left(\mathrm{N}_{\mathrm{Tx}}\right.$ and $\left.\mathrm{N}_{\mathrm{Rx}}\right)$, radii $r_{l}(\mathrm{~m})$ and $r_{2}(\mathrm{~m})$, permeability $\mu(\mathrm{H} / \mathrm{m})$ and the gap of the coil $d(\mathrm{~m})$ can be computed as Eq. 1.

$M=\frac{\mu \pi N_{T x} N_{R x} r_{1}^{2} r_{2}^{2}}{\left[\left(r_{1}+r_{2}\right)^{2}+d^{2}\right] \sqrt{\left(r_{1}+r_{2}\right)^{2}+d^{2}}}$

If coil inductance $(\mathrm{H})$ is $L_{T x}$ and $L_{R x}$, the magnetic coupling coefficient formula is as in Eq. 2:

$k=\frac{M}{\sqrt{L_{T x} \cdot L_{R x}}}$

\subsubsection{Flat Spiral Coil}

For Flat spiral coil [16], as shown in Figure 5, we can calculate L (Inductance), R (Resistivity), C (Capacitance), Q (Quality factor) value by considering the outside diameter (Do), number of turns (N), the distance between the windings (p) and the diameter of the wire (w), the inner diameter (Di), the average value of radius (a), length of wire (l) and the winding width (c) must also be considered, as illustrated in Figure 6 . All these quantities are in meters.

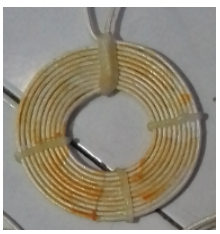

Figure 5: Flat spiral coil 


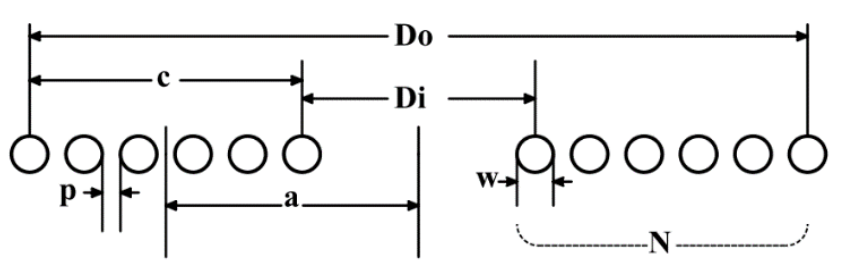

Figure 6: The cross-sectional view of flat spiral coil [16]

The Self-Inductance on this coil is obtained from the Wheeler's formula,

$L(H)=\frac{N^{2}\left(D_{o}-N(w+p)\right)^{2}}{16 D_{o}+28 N(w+p)} \times \frac{39.37}{10^{6}}$

The self-capacitance depends on the relative permittivity coil wire, the diameter of each coil, number of turns $\mathrm{N}$, and the distance between windings. Usually, the self-capacitance magnitude on the order of a few $\mathrm{pF}$ and this is very small when compared with the tuning capacitor for resonance IPT. It is, therefore, necessary tuning capacitance of a coil inductance and defined in terms of the resonant frequency as formulated as follows:

$C(F)=\frac{1}{(2 \pi f)^{2} L}$

Resistance:

$R_{D C}(O h m)=\frac{1}{\sigma \pi\left(\frac{w}{2}\right)^{2}}, \delta=\frac{1}{\sqrt{\pi f \sigma \mu_{0}}}$

$R=R_{D C} \frac{w}{4 \delta}=\sqrt{\frac{\pi f \mu_{0}}{\sigma}} \frac{N\left(D_{o}-N(w+p)\right)}{w}$

Quality Factor:

$Q=\frac{1}{R} \sqrt{\frac{L}{C}}=\frac{39,37}{10^{6}} \sqrt{\frac{f \pi \sigma}{\mu_{0}}} \frac{w N\left(D_{o}-N(w+p)\right)}{8 D_{o}+14 N(w+p)}$

This formula is used for optimization of the coil design for a high $\mathrm{Q}$ and ensure resonance at the desired operating frequency [8].

\subsubsection{Rectangular Coil}

In [14], Kuzey et al presents a formula for rectangleshaped coils, as given in Figure 7 with the following equation:

$r_{1}=\sqrt{\frac{N_{T x} S_{T x}}{\pi}} ; \quad r_{2}=\sqrt{\frac{N_{R x} S_{R x}}{\pi}}$

For the coil resistance's formula is as equation 9 where is $\rho_{c u}$ for copper, $\mathrm{a}_{1}$ and $\mathrm{b}_{1}$ is dimension of coil.

$R_{T x}=\rho_{c u} N_{T x} \frac{2\left(a_{1}+b_{1}\right)}{S_{T x}}$

To calculate the self-inductance of Tx and Rx coil, we used equation 10 and 11 :

$$
\begin{aligned}
& L_{T X}=\frac{\mu_{0}}{\pi} N_{T x}^{2}\left[a_{1} \ln \frac{2 a_{1} b_{1}}{r_{1}\left(a_{1}+\sqrt{\left(a_{1}^{2}+b_{1}^{2}\right)}\right)}+b_{1} \ln \frac{2 a_{1} b_{1}}{r_{1}\left(b_{1}+\sqrt{\left(a_{1}^{2}+b_{1}^{2}\right)}\right)}\right. \\
& -2\left(a_{1}+b_{1}-\sqrt{\left.a_{1}^{2}+b_{1}^{2}\right)}+0.25\left(a_{1}+b_{1}\right)\right] \\
& L_{R x}=\frac{\mu_{0}}{\pi} N_{R x}^{2}\left[a_{2} \ln \frac{2 a_{2} b_{2}}{r_{2}\left(a_{2}+\sqrt{\left(a_{2}^{2}+b_{2}^{2}\right)}\right)}+b_{2} \ln \frac{2 a_{2} b_{2}}{r_{2}\left(b_{2}+\sqrt{\left(a_{2}^{2}+b_{2}^{2}\right)}\right)}\right. \\
& -2\left(a_{2}+b_{2}-\sqrt{\left.a_{2}^{2}+b_{2}^{2}\right)}+0.25\left(a_{2}+b_{2}\right)\right]
\end{aligned}
$$

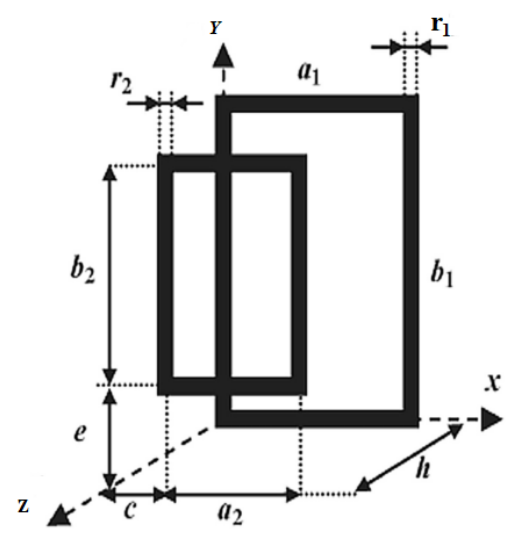

Figure 7: Rectangular Coil [14]

\subsection{Efficiency}

The efficiency of IPT depends on the quality factor of each coil Tx and Rx. The values of the quality factor can be calculated by assuming working independently for each coil. Moreover, the efficiency depends significantly on the tightness of the coupling between the coils, which is symbolized by the coupling coefficient $\mathrm{k}$ [9].

In theory, the maximum efficiency $\left(\mathrm{PTE}_{\max }\right)$ for IPT configuration that can be obtained can be expressed as a function of the quality factor of the coil $\left(\mathrm{Q}_{\mathrm{Tx}}\right.$ and $\left.\mathrm{Q}_{\mathrm{Rx}}\right)$, and the coupling coefficient $(\mathrm{k})$. PTE $\mathrm{Pax}_{\max }$ only characterized by the design of the coil (specify Q) and the relative geometry between coils (determine $\mathrm{k}$ ). Therefore, $\mathrm{PTE}_{\max }$ formula can be directly used for analysis coil design and geometry [10].

$P T E_{\text {max }}=\frac{k^{2} Q_{T x} Q_{R x}}{1+\sqrt{1+k^{2} Q_{T x} Q_{R x}}}$

The maximum system efficiency is obtained by optimal inductive coupling coefficient, appropriate compensation topology, and operating at the same resonant frequency $\left(f_{0}\right)$. The same resonant frequency can be achieved if the inductive reactance is equal to capacitive reactance so that the equivalent impedance is equal to the circuit resistance [14],

$f_{0}=\frac{1}{2 \pi \sqrt{L_{T x} C_{T x}}}=\frac{1}{2 \pi \sqrt{L_{R x} C_{R x}}}$

$\mathrm{L}_{\mathrm{Tx}}$ and $\mathrm{C}_{\mathrm{Tx}}$ is the equivalent value of the inductive reactance and capacitive reactance of the design on the transmitter coil while the $L_{R x}$ and $C_{R x}$ is equivalent to the value of the 
inductive reactance and capacitive reactance of the design on the receiver coil.

\subsection{Research Positioning}

In 2014, Benjamin $H$ Water et al [16] presented an equation for calculating the ratio and size of circle coils (equations 3 to 7). This equation is used to accurately calculate self-inductance, capacitance, resistance, quality factor in flat circle spiral coil. To validate the equation, Benjamin $H$ Water experimented with pitch variation and number of turns, of the same diameter as shown in Figure 8.

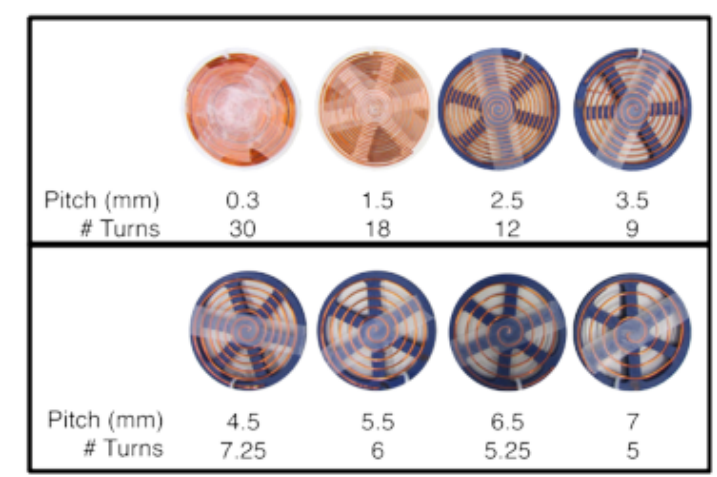

Figure 8: Variation of pitch distance and number of turns [16]

Budhia et al [10] explained that Circular couplers topology is the most commonly used in the literature; But essentially they have a limited coupling. Flux patterns they certainly limit the operational air gap and tolerance to misalignment. Budhia experimented by comparing the topology circle compared to the DD topology (Double D) which is double-lined circle topology, as well as with DDDDQ combined topology (double D-Quadrature Coil) which is adding rectangle coil to circle coil to solve coupling's problem. This test uses a distance between $20 \mathrm{~cm}$ coil and 20 $\mathrm{kHz}$ frequency. The result is the DD-DDQ topology can increase the charging area 5 times more than the circle topology for the same coil area.

Kalwar et al [4] used QDQ (Quad D Quadrature) to maintain a high coupling coefficient during a reasonable misalignment condition. The QDQ design uses 4 mutually adjacent circle coils and a rectangle coil and between the Tx and $\mathrm{Rx}$ coils is identical to capture the maximum flux in any position. This experiment uses a resonance frequency of 33 $\mathrm{kHz}$ and a distance between $15 \mathrm{~cm}$ coils. The coil design between $\mathrm{Tx}$ and $\mathrm{Rx}$ is made identical. Kalwar experiment for misalignment condition reaches a position $50 \%$ of the total area of the coil.

Kuzey et al [14] proposed coil design with rectangle shape but coil size Tx $60 \mathrm{~cm}$ x $100 \mathrm{~cm}$ and coil size Rx $60 \mathrm{~cm}$ x 60 $\mathrm{cm}$ with resonance frequency $17.702 \mathrm{kHz}$ and the distance between coil $20 \mathrm{~cm}$ as in Figure 7. Kuzey also conducts tests for angular misalignment conditions.
Zhichao Luo [17] compared the performance between square coils with circular coils. Both coils are made with the same parameters. A square coil with dimensions of $600 \mathrm{~mm}$ x $600 \mathrm{~mm}$, whereas a circular coil is made with a radius of $300 \mathrm{~mm}$ or a diameter of $600 \mathrm{~mm}$, other parameters such as pitch, wire diameter, number of turns is made equal. The resulting square-shaped coil produces mutual inductance and coupling coefficients higher than the circular coil so that the rectangular coils prove to be a better choice for WPT systems.

\section{Proposed Coil Geometry Design}

This paper used three coil designs. We redesigned the coil structure given by the reference [14] with identical Tx and $\mathrm{Rx}$ coils and called it $1^{\text {st }}$ design. The $2^{\text {nd }}$ design is a redesign of the coil structure as given by reference [4], whereas the $3^{\text {rd }}$ design is a new coil structure proposed in this work. All of design as illustrated in Figure 9.
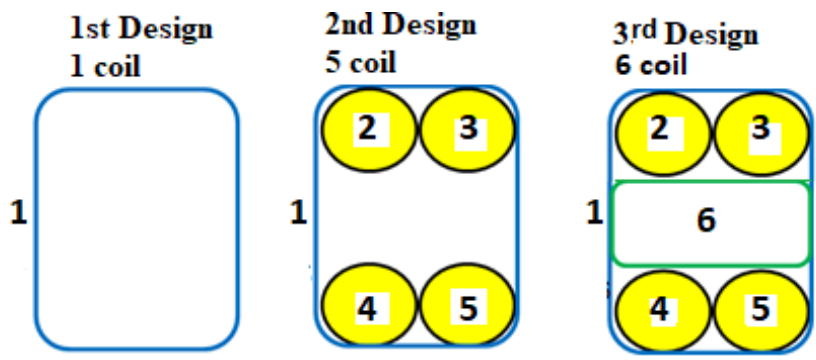

Figure 9: Coil Design Geometry

Coil number 1 is made with dimensions of length and width, for the outer dimension is $36.5 \mathrm{~cm} \times 26.5 \mathrm{~cm}$, while the inner dimension is $30 \mathrm{~cm} \times 20 \mathrm{~cm}$. For $2,3,4$ and 5 coils are made identically with an outer diameter of $9.8 \mathrm{~cm}$ and an inner diameter of $4.74 \mathrm{~cm}$. While for the outer dimension coil number 6 is $10 \mathrm{~cm} \times 20 \mathrm{~cm}$. All coils are made with the same litz wire with diameter of wire $1.15 \mathrm{~mm}$ and number of turns each coils is 10 turns. All of these coil designs are tested with a maximum power of 4.6 watts. All of coils parameter data gived in table 1 .

Table 1: Coil Dimension

\begin{tabular}{|lrl|}
\hline Max power & 4.6 & watt \\
\hline Number of strand & 32 & pcs \\
Diameter of strand & 0.19 & $\mathrm{~mm}$ \\
Total wire diameter (w) & 1.15 & $\mathrm{~mm}$ \\
Total wire diameter + Insulation & 2.53 & $\mathrm{~mm}$ \\
The distance between wires (p) & 1.38 & $\mathrm{~mm}$ \\
Wire + Insulation's Area (Sx ; S $\mathrm{S}_{\mathrm{Rx})}$ & 5.024 & $\mathrm{~mm}^{2}$ \\
\hline \multicolumn{1}{|c}{ Coil 1 } & & \\
\hline & 36.5 & $\mathrm{~cm}$ \\
\hline Outer length & 26.5 & $\mathrm{~cm}$ \\
Outer width & 30 & $\mathrm{~cm}$ \\
Inner length & 20 & $\mathrm{~cm}$ \\
Inner width & 10 & \\
Number of turn $(\mathrm{N})$ & &
\end{tabular}




\begin{tabular}{|c|c|c|}
\hline \multicolumn{3}{|c|}{ Coil $2=$ Coil $3=$ Coil $4=$ Coil 5} \\
\hline Outer diameter (D0) & 9.8 & $\mathrm{~cm}$ \\
\hline Inner diameter (Di) & 4.74 & $\mathrm{~cm}$ \\
\hline Number of turn $(\mathrm{N})$ & 10 & \\
\hline \multicolumn{3}{|c|}{ Coil 6} \\
\hline Outer length & 20 & $\mathrm{~cm}$ \\
\hline Outer width & 10 & $\mathrm{~cm}$ \\
\hline Number of turn $(\mathrm{N})$ & 10 & \\
\hline
\end{tabular}

In this work a rectangular coil (coil number 6) was added to the middle of the previous design to increase the power transfer performance at a misalignment condition. This is based on [4] which states that the rectangular coil have a transfer performance better than circle coil at misalignment condition.

From the equation (3), (8), (10) and (11), the calculation of the inductance value in coil number 1 is $86.126 \mu \mathrm{H}$, coil number 2,3,4,5 each has the value $9.141 \mathrm{uH}$, for coil number 6 the value of $\mathrm{L}$ is $34.555 \mu \mathrm{H}$ so the total of the inductance is $157.245 \mu \mathrm{H}$. The total inductance of the measurement is 150 $\mu \mathrm{H}$. The results of the inductance calculation and the $\mathrm{C}$ value used, can produce a resonance frequency of $25.619 \mathrm{kHz}$ on the Tx coil, and on the Rx circuit arranged with the same frequency.

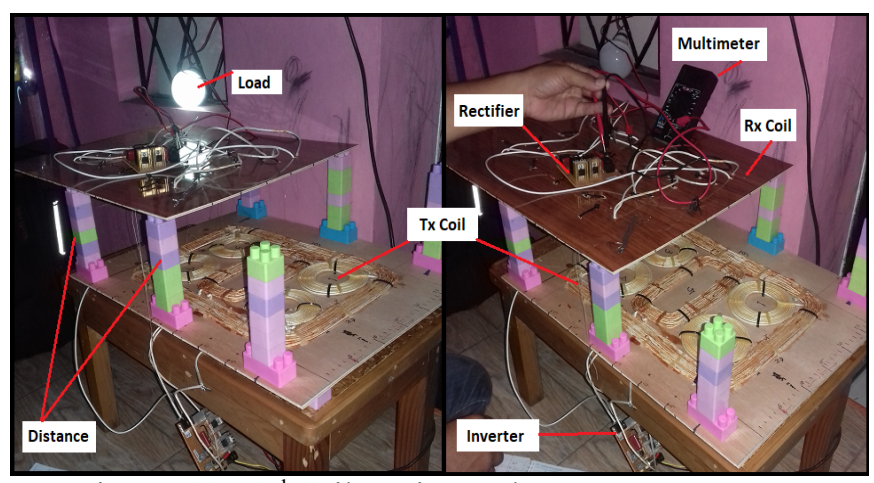

Figure 10: $3^{\text {rd }}$ Coil Design and Measurement

Figure 10 shows the full inductive power transfer systems built in this research. The measurement gives a periodic signal with the fundamental frequency of $35.088 \mathrm{kHz}$ produced by the inverter. This frequency is above the resonance frequency of coil structure. Behind the Rx coil, the $\mathrm{AC}$ voltage is converted to $\mathrm{DC}$ with a rectifier. Measurement of efficiency in this work is carried out by comparing the DC output power at load to the DC input power. The result of determining the amount of load impedance obtained by measuring the output stage in open circuit condition and the current in short circuit condition at load point.

It is expected that the inverter can be used for all coil designs, all fixed coil used in an attempt to meet the value of the inductor L2 and L3 respectively $150 \mu \mathrm{H}$ as shown in Figure 11. On the $1^{\text {st }}$ of design, there is only one coil used for power transfer process, therefore the other coil remains installed and functioning as a resonator. Likewise with the $2^{\text {nd }}$ design, when only 5 coils are used for the power transfer process, 1 remaining coil is still installed as a resonator.

In this research, all the coils were installed in series electrically.

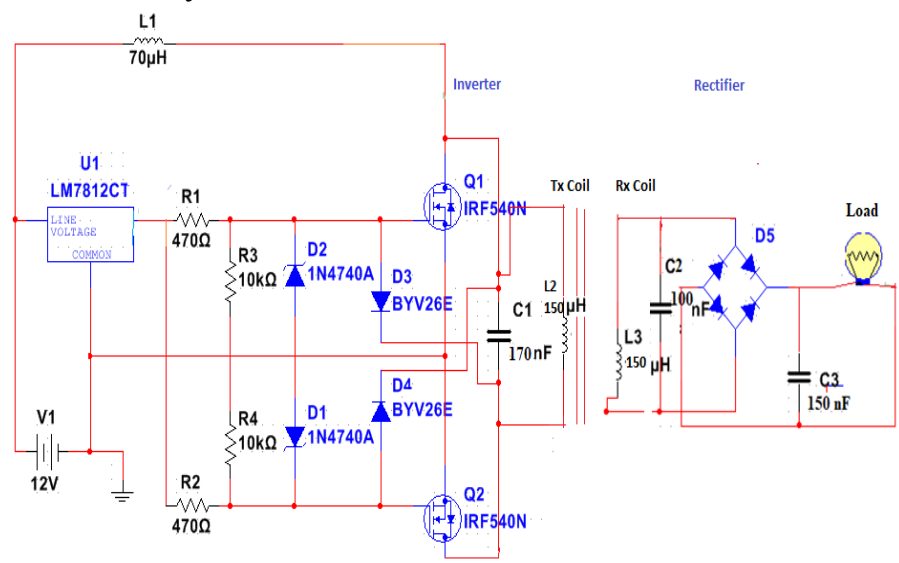

Figure 11: Full circuit schematic of the research

\section{Experiment Result}

Table 2 and Figure 12 gives the efficiency of the power transfer as function of distance at a perfect alignment. It is found that at the coil distance of $2.8 \mathrm{~cm}$ to $13.9 \mathrm{~cm}$ the efficiency of $3^{\text {rd }}$ design is better than the two other designs from the highest value of $48.9 \%$ and decreases to $16.5 \%$. The declining value of efficiency due to the distance coil increased fit to equation (1) (2) and (12) where the mutual inductance is inversely proportional to the distance of the coil, magnetic coupling coefficient proportional to the mutual inductance and PTE $_{\max }$ proportional to magnetic coupling coefficient so higher mutual inductance values cause increased efficiency.

Table 2: Efficiency at a Perfect alignment

\begin{tabular}{|r|r|r|r|}
\hline $\begin{array}{c}\text { Coil } \\
\text { Distance } \\
\text { (cm) }\end{array}$ & $\begin{array}{c}\text { 1st } \\
\text { Design }\end{array}$ & $\begin{array}{c}\text { 2nd } \\
\text { Design }\end{array}$ & $\begin{array}{c}\text { 3rd } \\
\text { Design }\end{array}$ \\
\hline \hline 2.8 & $44.9 \%$ & $47.4 \%$ & $48.9 \%$ \\
\hline 4.6 & $45.8 \%$ & $42.0 \%$ & $48.4 \%$ \\
\hline 6.5 & $35.6 \%$ & $37.0 \%$ & $41.2 \%$ \\
\hline 8.4 & $27.6 \%$ & $30.7 \%$ & $35.2 \%$ \\
\hline 10.3 & $22.1 \%$ & $22.9 \%$ & $26.9 \%$ \\
\hline 12.2 & $15.5 \%$ & $19.7 \%$ & $22.3 \%$ \\
\hline 13.9 & $11.1 \%$ & $15.6 \%$ & $16.5 \%$ \\
\hline
\end{tabular}

At the misalignment conditions (X-axis displacement), Figure 13 shown that the misalignment between $0 \mathrm{~cm}$ up to $10 \mathrm{~cm}$ (misalignment of $0 \%-27.4 \%$ ) for a coil distance of 2.8 $\mathrm{cm}, 4.6 \mathrm{~cm}, 6.5 \mathrm{~cm}$ and $8.4 \mathrm{~cm}$, the $3^{\text {rd }}$ design produces the highest efficiency and its value coincides with the $2^{\text {nd }}$ design when the coil distance is more than $10.3 \mathrm{~cm}$. In a X-axis displacement condition of more than $10.3 \mathrm{~cm}$, the efficiency of the $3^{\text {rd }}$ design decreases as it is influenced by coil number 
6 on Tx parallel to coil number 2 and 3 in $R x$, where there is a different magnetic field direction so the resultant drop.

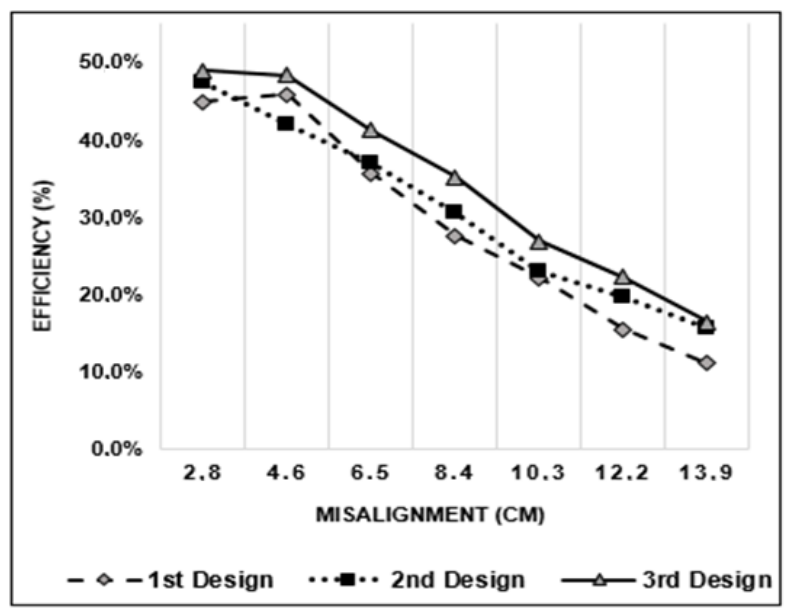

Figure 12: Efficiency at a perfect alignment

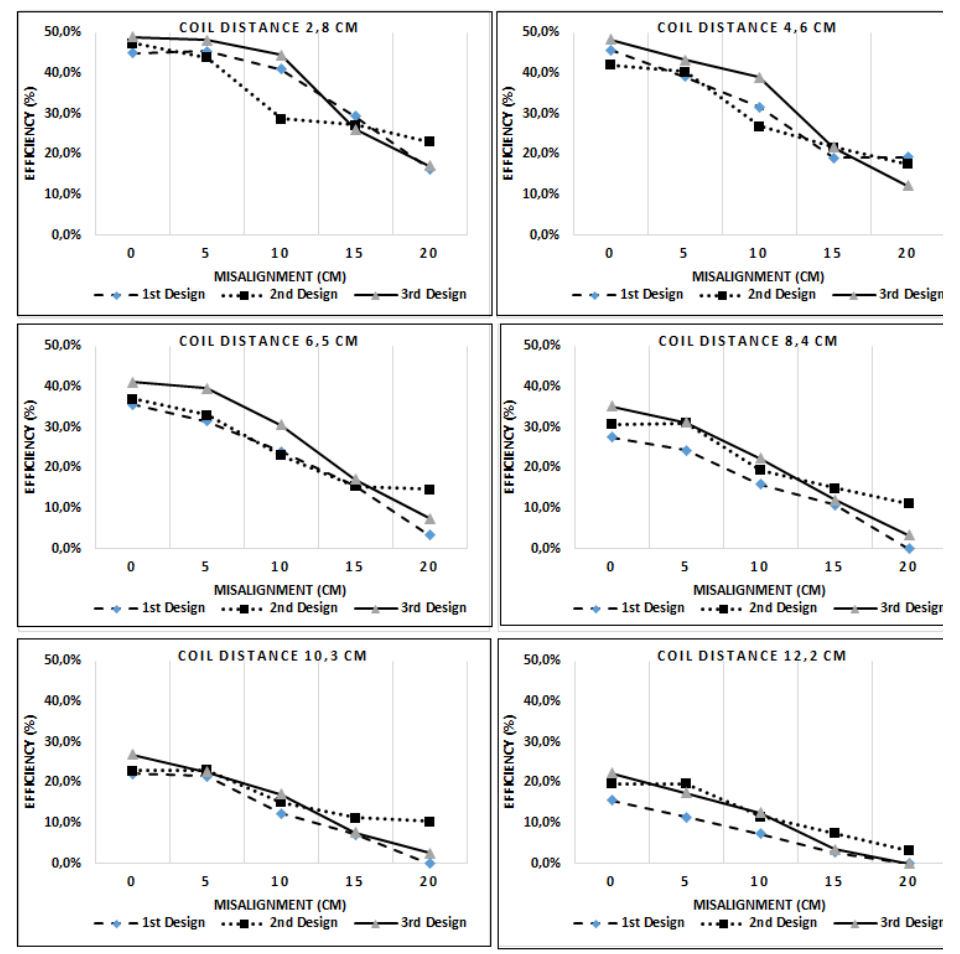

Figure 13: Efficiency for several $\mathrm{X}$-axis displacements

\section{Conclusions and Future Work}

Comparison between the results of three coil designs gave, generally for perfect alignment conditions, $3^{\text {rd }}$ design is the best from others for all coil spacing. While the misalignment up to $10 \mathrm{~cm}(27.4 \%)$, the $3^{\text {rd }}$ design performance still shows the best value up to the coil distance of $10.3 \mathrm{~cm}$. At a coil distance above $10.3 \mathrm{~cm}$ and a misalignment above $10 \mathrm{~cm}$, the efficiency of $3^{\text {rd }}$ design slightly decreases below $2^{\text {nd }}$ design, but is still high compared to $1^{\text {st }}$ design.

\section{References}

L. Zhao, D. Thrimawithana, and U. Madawala, "A Hybrid Bi-directional Wireless EV Charging System Tolerant to Pad Misalignment," IEEE Trans. Ind. Electron., vol. 64, no. 99, pp. 7079-7086, 2017.

K. Fotopoulou and B. W. Flynn, "Wireless Power Transfer in Loosely Coupled Links: Coil Misalignment Model," IEEE Journals Mag., vol. 47, no. 2, pp. 416-430, 2011.

$X$. Mou and H. Sun, "Wireless Power Transfer : Survey and Roadmap," in 2015 IEEE 81st Vehicular Technology Conference (VTC Spring), 2015, no. 2, pp. 1-5.

K. A. Kalwar, S. Mekhilef, M. Seyedmahmoudian, and B. Horan, "Coil Design for High Misalignment Tolerant Inductive Power Transfer System for EV Charging," MDPI (Multidisciplinary Digit. Publ. Institute)- Energies, vol. 9, no. 11, pp. 12-17, 2016. M. L. Ferraro, "Design and Control of Inductive Power Transfer System for Electric Vehicle Charging," Universite de Toulouse, Laplace,France, 2017.

X. Wei, Z. Wang, and H. Dai, "A Critical Review of Wireless Power Transfer via Strongly Coupled Magnetic Resonances," MDPI (Multidisciplinary Digit. Publ. Institute)- Energies, vol. 7, no. 7, pp. 4316-4341, 2014.

C. Carretero, "Coupling Power Losses in Inductive Power Transfer Systems with Litz-Wire Coils," IEEE Trans. Ind. Electron., vol. 64, no. 6, pp. 4474-4482, 2017.

X. Lu, P. Wang, D. Niyato, D. I. Kim, Z. Han, and C. Engineering, "Wireless Charging Technologies: Fundamentals , Standards , and Network Applications," IEEE Commun. Surv. Tutorials, vol. 18 , no. 2, pp. 1413-1452, 2016.

[9] Lu,Xiao, D. Nyato, P. Wang, D. In Kim, and Z. Han, "Wireless Charger Networking For Mobile Device: Fundamentals,Standards, and Applications," IEEE Wirel. Commun., vol. 22, no. 2, pp. 126-135, 2015.

[10] M. Budhia, J. T. Boys, and G. A. Covic, "Development of a Single-Sided Flux Magnetic Coupler for Electric Vehicle IPT Charging Systems," IEEE Trans. Ind. Electron., vol. 60, no. 1, pp. 318328, 2013.

[11] X. Qu, Y. Jing, H. Han, S. C. Wong, and C. K. Tse, 
"Higher Order Compensation for Inductive-PowerTransfer Converters with Constant-Voltage or Constant-Current Output Combating Transformer Parameter Constraints," IEEE Trans. Power Electron., vol. 32, no. 1, pp. 394-405, 2017.

[12] M. Dehghanian, A. Namadmalan, and $M$. Saradarzadeh, "Optimum Design for Series-Series Compensated Inductive Power Transfer Systems," in 8th Power Electronics, Drive Systems \& Technologies Conference (PEDSTC 2017), 2017, no. 2.

[13] P. Raval, D. Kacprzak, and A. Hu, "Computational Finite Element Software Assisted Development of a 3D Inductively Coupled Power Transfer System," Adv. Electromagn., vol. 2, no. 3, pp. 11-17, 2013.

[14] S. Kuzey, S. Balci, and N. Altin, "Design and analysis of a wireless power transfer system with alignment errors for electrical vehicle applications," Int. J. Hydrog. Energy xxx Elsevier, pp. 1-12, 2017.

[15] C. Nataraj, S. Khan, M. H. Habaebi, and A. G. A. Muthalif, "Analysis of Mutual Inductance and Coupling Factor of," ARPN J. Eng. Appl. Sci., vol. 12, no. 13, pp. 4007-4012, 2017.

[16] B. H. Waters, B. J. Mahoney, G. Lee, and J. R. Smith, "Optimal Coil Size Ratios for Wireless Power Transfer Applications," in IEEE International Symposium on Circuits and Systems (ISCAS), 2014, vol. 1, no. 1, pp. 2045-2048.

[17] Z. Luo and X. Wei, "Analysis of Square and Circular Planar Spiral Coils in Wireless Power Transfer System for Electric Vehicles," IEEE Trans. Ind. Electron., vol. 65, no. 1, pp. 331-341, 2018. 